Printed in the United Kingdom (c) 1999 Cambridge Philosophical Society

\title{
Fractional integration, differentiation, and weighted Bergman spaces
}

\author{
By STEPHEN M. BUCKLEY $\dagger$ \\ Department of Mathematics, National University of Ireland, Maynooth, \\ Co. Kildare, Ireland \\ e-mail: sbuckley@maths . may . ie \\ PEKKA KOSKELA $†$ \\ Department of Mathematics, University of Jyväskylä, P.O. Box 35, \\ Fin-40351 Jyväskylä, Finland \\ e-mail: pkoskela@math.jyu.fi \\ AND DRAGAN VUKOTIĆ \\ Departamento de Matemáticas, Universidad Autónoma de Madrid, \\ Ciudad Universitaria de Cantoblanco, 28049 Madrid, Spain \\ e-mail: dragan.vukotic@uam.es
}

(Received 27 May 1997)

\section{Abstract}

We study the action of fractional differentiation and integration on weighted Bergman spaces and also the Taylor coefficients of functions in certain subclasses of these spaces. We then derive several criteria for the multipliers between such spaces, complementing and extending various recent results. Univalent Bergman functions are also considered.

\section{Introduction}

The question of describing the coefficient multipliers between various spaces of analytic functions is an old subject that essentially began with the works of Hardy and Littlewood. Their second paper on fractional integrals [16] in the 1930s contains several results of this kind, even though they do not explicitly mention multipliers. Results involving the integration or differentiation of an analytic function in the unit disc, such as the classical theorem of Littlewood and Paley (see [3] or [23]) which says that if $f \in H^{p}(p \geqslant 2)$ then $f^{\prime}$ belongs to the weighted Bergman space $A_{p-1}^{p}$, can be viewed as coefficient multiplier results. Some similar results for $p<2$ were obtained later in $[\mathbf{3}, \mathbf{1 7}, \mathbf{3 1}]$. A related problem also arises in connection with the differentiation operators and Carleson measures (area version): for which positive

$\dagger$ The first author was partially supported by NSF Grant DMS-9207715 and by Forbairt. The second author was partially supported by NSF Grant DMS-9305742 and by the Academy of Finland. 
values of $p, q$, and for which $\alpha, \beta>-1$, are the quantities

$$
\int_{\mathbf{D}}|f(z)-f(0)|^{p}\left(1-|z|^{2}\right)^{\alpha} d A(z) \text { and } \int_{\mathbf{D}}\left|f^{\prime}(z)\right|^{q}\left(1-|z|^{2}\right)^{\beta} d A(z)
$$

equivalent? Flett $([\mathbf{1 4}, \mathbf{1 5}])$ provided the answer in various cases; general questions of this type were also studied recently by Luecking [22]. A complete answer to this question follows from Theorem 1.3 below (which treats arbitrary fractional derivatives).

From the late 1950 s to the early 1970 s Flett ([13-15], etc.) obtained a number of precise results about the order of growth of a function and its (fractional) integrals and derivatives in terms of the Taylor series coefficients of functions using the general spaces $H(p, q, s)$. Since then, many authors have worked on the theory of multipliers between various spaces of analytic functions. The survey article by Campbell and Leach [9] and a recent paper of Blasco [6] contain many theorems and references. Some other related results can also be found in $[1,4,11,12,19,21,25,26]$.

Pavlović [28] found a characterization of $\left(A^{p}, A^{q}\right)$ for $0<p \leqslant 1, p \leqslant q<\infty$, in terms of the growth of integral means of a certain function associated with the 'candidate' sequence; here, $A^{p}$ is analytic $L^{p}(\mathbf{D})$ and $\left(A^{p}, A^{q}\right)$ is the space of multipliers from $A^{p}$ to $A^{q}$. Since it is not easy to control the growth of a function in terms of its coefficients (see the results in Section 4 on random changes of sign), it is of interest to find necessary or sufficient sequential conditions even in these cases.

In a significant breakthrough, Wojtaszczyk [30] characterized the multipliers $\left(A^{p}, A^{q}\right), 0<q \leqslant 2 \leqslant p<\infty$, in terms of mixed-norm sequence spaces $l^{\infty, r}$; this result was simplified and generalized by Anderson [2], Jevtić and Jovanović [18], and Blasco [6]. Blasco also described $\left(A^{p}, A^{2}\right)$ for $0<p \leqslant 1$. Vukotic [29] had earlier characterized $\left(A^{1}, A^{2}\right)$ and had given several conditions which are either necessary or sufficient for membership of $\left(A^{p}, A^{q}\right)$ in certain cases such as $0<p \leqslant 2 \leqslant q<\infty$.

In Section 1 we reduce the study of multipliers between weighted Bergman spaces to that for unweighted ones; this reduction, which we deduce from the work of Flett, appears to have been previously unnoticed.

One cannot characterize Bergman functions (for $p \neq 2$ ) in terms of the size of the Taylor coefficients but, in Section 2, we show that this is possible for functions with lacunary or monotonic Taylor sequences (these examples are important for later results).

In Section 3 we discuss the self-multipliers $(X, X)$ for certain spaces $X$ of analytic functions. We show that $(X, X)$ often contains $B V$, the sequences of bounded variation. Even when this is false, we can almost always say that $(X, X)$ contains the 'Taylor form' sequences (which form a subclass of $B V$ ); see Section 3 for precise statements.

A careful inspection of the literature reveals a need to justify the free interchange between two forms of fractional integrals and derivatives (those expressed in terms of the special function $B$ and those given by powers of $n$ ). This was done in certain cases by Flett [15]; Taylor form multipliers allow us to to do so for many function spaces.

In Section 4, we give various (but separate) necessary and sufficient conditions for a sequence to be a multiplier between weighted Bergman spaces. Finally, in Section 5, 
we briefly consider the action of fractional differentiation on univalent $A^{p}$ functions; in particular, we generalize part of a well-known result of Brennan [7].

\section{Preliminaries. Fractional derivatives and integrals}

Unless otherwise stated, analytic functions are assumed to be analytic on the unit disc $\mathbf{D} \subset \mathbf{C}$ and we identify such functions with their sequence of Taylor coefficients at the origin. Writing $d A$ for Lebesgue measure on $\mathbf{D}$, we are mainly interested in the weighted Bergman spaces $A_{\alpha}^{p}(0<p<\infty,-1<\alpha)$ of analytic functions $f$ for which

$$
\|f\|_{A_{\alpha}^{p}} \equiv\left(\int_{\mathbf{D}}|f(z)|^{p}(1-|z|)^{\alpha} d A(z)\right)^{1 / p}<\infty .
$$

We write $A^{p}=A_{0}^{p}$. We shall also use the more general spaces $H(p, q, s)$. Specifically if $0<p \leqslant \infty, 0<q, s<\infty$, this is defined as the space of analytic functions for which

$$
\|f\|_{H(p, q, s)} \equiv\left(\int_{0}^{1} M_{p}(r, f)^{q}(1-r)^{s q-1} d r\right)^{1 / q}<\infty,
$$

where $M_{p}(r, f)$ is the $L^{p}(\{z:|z|=r\})$ 'norm' of $f$. We also define $H(p, \infty, s) \equiv H_{s}^{p}$ for all $0<p \leqslant \infty, 0 \leqslant s$, to be the space of analytic functions for which

$$
\|f\|_{H_{s}^{p}} \equiv \sup _{0<r<1}(1-r)^{s} M_{p}(r, f)<\infty .
$$

It is clear that $A_{\alpha}^{p}=H(p, p,(1+\alpha) / p)$ and, in particular, $A^{p}=H(p, p, 1 / p)$. By an 'allowable' triple $(p, q, s)$, we shall simply mean a triple for which $H(p, q, s)$ has been defined above. To avoid cluttered lists of assumptions, we implicitly assume that all triples are allowable when discussing $H(p, q, s)$ spaces; similarly we implicitly assume that $0<p<\infty$ and $-1<\alpha<\infty$ for $A_{\alpha}^{p}$ spaces.

We say $\lambda=\left(\lambda_{n}\right)_{n=0}^{\infty}$ is a multiplier between the sequence spaces $X$ and $Y$, written $\lambda \in(X, Y)$, if $\lambda\left(a_{n}\right) \equiv\left(\lambda_{n} a_{n}\right) \in Y$ whenever $\left(a_{n}\right) \in X$. We define the fractional derivative multipliers by $D^{t}=\left(n^{t}\right)$ for all $t \in \mathbf{R}$. Although notationally unnecessary, we also define the fractional integral multipliers by $I^{t}=D^{-t}$. Here and later $0^{t}$ is taken to mean 0 (even if $t$ is negative); this abuse of arithmetic is harmless, because membership of any space considered below is always an asymptotic property.

Given any sequence $a=\left(a_{k}\right)$, and any $0<p \leqslant \infty$, we define the sequence $s(a, p)=$ $\left(s_{n}(a, p)\right)_{n=0}^{\infty}$ by $s_{n}(a, p)=\left(\sum_{k=2^{n}}^{2^{n+1}-1}\left|a_{k}\right|^{p}\right)^{1 / p}$ (with the usual alteration for $\left.p=\infty\right)$. If also $0<q \leqslant \infty$, the space $l(p, q)$ is defined as the set of all sequences $a$ for which $s(a, p) \in l^{q}$. Clearly $l\left(p_{1}, q_{1}\right) \subseteq l\left(p_{2}, q_{2}\right)$ if $p_{1} \leqslant p_{2}, q_{1} \leqslant q_{2}$, and also $l(p, p)=l^{p}$ for all $p>0$. In general the $l(p, q)$ condition can be viewed as a slight perturbation of the $l^{p}$ condition (for instance, if $q<\infty,\left(n^{-t}\right) \in l(p, q)$ if and only if $t p>1$ ).

If $X$ is a sequence space and, for every sequence $\left(a_{n}\right), \lambda\left(a_{n}\right) \in X$ if and only if $\lambda^{\prime}\left(a_{n}\right) \in X$, we say that $\lambda$ and $\lambda^{\prime}$ are equivalent on $X$. We simply say that $\lambda$ and $\lambda^{\prime}$ are equivalent if they are equivalent on all weighted Bergman spaces; obviously, equivalent sequences are multipliers between the same pairs of Bergman spaces. If $A$ and $B$ are any positive quantities, we write $A \approx B$ if $A / C \leqslant B \leqslant C A$, where $C$ is any positive constant (whose exact value does not matter).

We first gather together some known results for the spaces $H(p, q, s)$ and $A_{\alpha}^{p}$ in a pair of lemmas. Parts (i)-(iii) of our first lemma (see [6]) are fairly elementary; (iv) 
is deeper and was proved by Flett ([14] for $s=0, q=\infty$ and [15, theorem 6$]$ for all other cases). Part (v) follows easily by rewriting theorem 5(iii) of [15]. The second lemma is taken from [27] (parts (i)-(iv), (vi)), while part (v) is from [17].

Lemma 1-1. If $p, q, s, s+t>0, p_{1} \leqslant p_{2}, q_{1} \leqslant q_{2}$, and $0<s_{1} \leqslant s_{2}$, then

(i) $H^{p_{2}} \subset H\left(p_{2}, q_{1}, s_{1}\right) \subset H\left(p_{1}, q_{2}, s_{2}\right) \subset H_{s_{2}}^{p_{1}}$;

(ii) $H\left(p_{1}, q, s\right) \subset H\left(p_{2}, q, s+p_{1}^{-1}-p_{2}^{-1}\right)$;

(iii) $H\left(p, q_{2}, s\right) \subset H\left(p, q_{1}, s+q_{1}^{-1}-q_{2}^{-1}\right)$;

(iv) $D^{t} \in(H(p, q, s), H(p, q, s+t))$;

(v) $I^{s} \in\left(H(p, p, s), H^{p}\right)$, for $0<p \leqslant 2$.

Lemma 1.2. Let $f(z)=\sum_{n=0}^{\infty} a_{n} z^{n}$.

(i) If $0<p \leqslant 1$ and $f(z) \in A_{\alpha}^{p}$, then $a_{n}=o\left(n^{\frac{\alpha+2}{p}-1}\right)$.

(ii) If $1 \leqslant p<\infty$ and $f(z) \in A_{\alpha}^{p}$, then $a_{n}=o\left(n^{\frac{\alpha+1}{p}}\right)$.

(iii) If $f(z) \in A_{\alpha}^{p}$, then $\sum_{n=1}^{\infty} n^{p-\alpha-3}\left|a_{n}\right|^{p}<\infty$ whenever $0<p \leqslant 2$, and the converse holds whenever $p \geqslant 2$.

(iv) If $f(z) \in A_{\alpha}^{p}$, then $\sum_{n=1}^{\infty} n^{-\alpha-1}\left|a_{n}\right|^{p}<\infty$ whenever $p \geqslant 2$, and the converse holds whenever $0<p \leqslant 2$.

(v) Suppose $p, p^{\prime}$ are conjugate indices. If $f(z) \in A^{p}$, then $\sum_{n=1}^{\infty} n^{1-p^{\prime}}\left|a_{n}\right|^{p^{\prime}}<\infty$ whenever $1<p \leqslant 2$, and the converse holds whenever $p \geqslant 2$.

(vi) If $q<p$, then $A_{\alpha}^{p} \subset A_{\beta}^{q}$ for all $\beta$ satisfying $(\beta+1) q^{-1}>(\alpha+1) p^{-1}$.

We now give an answer to the question on equivalence of two integrals mentioned in the introduction. The next couple of results follow easily from Lemmas 1.1 and $1 \cdot 2$, but do not seem to have been noticed or written down before in full generality. In particular, they generalize earlier results of Flett [15]. We would like to stress that parts $(c)$ and $(d)$ of Corollary 1.4 completely reduce the study of multipliers and coefficient estimates on weighted Bergman spaces to the unweighted case. All indices and inequalities in these first two results are sharp, and one cannot replace the ' $<$ ' by ' $\leqslant$ ' in parts $(b)$ of Theorem 1.3 and Corollary 1.4, but we postpone justifications of sharpness until later (see comments after Proposition 2.1 and Lemma 3.8).

Theorem 1·3. If $-1<\alpha, \beta<\infty, t \in \mathbf{R}$, and $0<p, q \leqslant \infty$, then $D^{t} \in\left(A_{\alpha}^{p}, A_{\beta}^{q}\right)$ (a) if $p \leqslant q$ and $(2+\beta) q^{-1} \geqslant(2+\alpha) p^{-1}+t$, or (b) if $p>q$ and $(1+\beta) q^{-1}>(1+\alpha) p^{-1}+t$.

Proof. The special case $p=q$ of Lemma 1.1(iv) gives $D^{t} \in\left(A_{\alpha}^{p}, A_{\alpha+t p}^{p}\right)$ for all $\alpha>-1, t \in \mathbf{R}, p>0$. But by Lemma $1 \cdot 1($ ii),

$$
A_{\alpha+t p}^{p}=H\left(p, p,(1+\alpha) p^{-1}+t\right) \subset H\left(q, q,(2+\alpha) p^{-1}-q^{-1}+t\right)=A_{\beta}^{q}
$$

for any $q \geqslant p$, giving $(a)$. Similarly, (b) follows by using Lemmas $1 \cdot 1$ (iv) and $1 \cdot 2(\mathrm{vi})$.

Corollary $1 \cdot 4$.

(a) If $p \leqslant q$, and $t \geqslant(2+\alpha)\left(p^{-1}-q^{-1}\right)$, then $I^{t} \in\left(A_{\alpha}^{p}, A_{\alpha}^{q}\right)$.

(b) If $p>q$, and $t<(1+\alpha)\left(q^{-1}-p^{-1}\right)$, then $D^{t} \in\left(A_{\alpha}^{p}, A_{\alpha}^{q}\right)$.

(c) $\left(a_{n}\right) \in A_{\alpha}^{p}$ if and only in $\left(n^{-\alpha / p} a_{n}\right) \in A^{p}$.

(d) $\left(\lambda_{n}\right) \in\left(A_{\alpha}^{p}, A_{\beta}^{q}\right)$ if and only if $\left(n^{\alpha p^{-1}-\beta q^{-1}} \lambda_{n}\right) \in\left(A^{p}, A^{q}\right)$.

Proof. $(a)$ and $(b)$ are special cases of the corresponding parts of Theorem 1.3; $(c)$ follows immediately from $(a)$ and $(d)$ from $(c)$. 
Fractional integration, differentiation, and weighted Bergman spaces 373

Since the indices in Theorem 1.3 are best possible (as we shall see), the case $t=0$ gives the best possible imbeddings of the form $A_{\alpha}^{p} \subset A_{\beta}^{q}$. With one exception, these are as given in theorem 1 of [27]. The exception is their imbedding $3^{\circ}$ (and the equivalent $\left.7^{\circ}\right)$ which says that if $(1+\alpha) / p>(1+\beta) / q$ and $(2+\alpha) / p<(2+\beta) / q$ then $A_{\alpha}^{p} \subset A_{\beta+1}^{q}$. In this case, we must have $p>q$ and so Theorem 1.3(b) tells us that $A_{\alpha}^{p} \subset A_{\gamma}^{q}$ for all $\gamma>\gamma_{0}=(1+\alpha) q p^{-1}-1$. Note that $\beta<\gamma_{0}<\beta+1$ in all cases.

Suppose $f, g$ have Taylor coefficients $\left(a_{n}\right),\left(b_{n}\right)$ respectively. We shall make use of the pairing

$$
<f, g>=\frac{1}{\pi} \int_{\mathbf{D}} f \bar{g} d A=\sum_{n=0}^{\infty} \frac{a_{n} \overline{b_{n}}}{n+1} .
$$

Suppose $1<p<\infty$. It is well known (see, for example, theorem 1.16 of [5]) that the dual $\left(A^{p}\right)^{*}$ of $A^{p}$ with respect to this pairing is $A^{p^{\prime}}$ where $p^{\prime}=p /(p-1)$ is the index dual to $p$; furthermore the $\left(A^{p}\right)^{*}$-norm of the linear functional corresponding to $g \in A^{p^{\prime}}$ is comparable with $\|g\|_{A^{p^{\prime}}}$. If also $1<q<\infty$ and $q^{\prime}=q /(q-1)$, it follows that $\lambda \in\left(A^{p}, A^{q}\right)$ if and only if $\bar{\lambda} \in\left(A^{q^{\prime}}, A^{p^{\prime}}\right)$. But it is clear that $\left(a_{n}\right) \in A^{r}$ if and only if $\left(\overline{a_{n}}\right) \in A^{r}(0<r<\infty)$, and so we deduce that $\left(A^{p}, A^{q}\right)=\left(A^{q^{\prime}}, A^{p^{\prime}}\right)$, a useful duality result that we shall use later in this paper. It also follows by duality (although now the pairing $\sum_{n=0}^{\infty} a_{n} \overline{b_{n}}$ is more natural) that $\left(H^{p}, H^{q}\right)=\left(H^{q^{\prime}}, H^{p^{\prime}}\right)$.

\section{Functions with lacunary or monotonic Taylor coefficients}

We shall later look at necessary and sufficient conditions for multipliers between Bergman spaces. For this analysis, it will be useful to build up a stock of examples and non-examples of functions in $A_{\alpha}^{p}$.

Our first class of examples are lacunary sequences (recall that we are identifying analytic functions with their sequences of Taylor coefficients). We define the $k$ th dyadic block $B_{k}$ to be the set of integers from $2^{k-1}$ to $2^{k}-1$ inclusive, if $k>0$, and $B_{0}=\{0\}$. Also let $\widetilde{B}_{k}$ be the larger set of integers from $2^{k-2}+1$ to $2^{k}-1$ inclusive, if $k>1$, and $\widetilde{B}_{0}=\{0\}, \widetilde{B}_{1}=\{0,1\}$. Our lacunary sequences include all the usual lacunary $\left(a_{n}\right)$, where $a_{n}=0$ if $n$ is not a power of 2 but, in view of our later needs, we define a lacunary sequence to be any sequence which has at most one non-zero term with index in any one block $B_{k}$ (and hence at most two non-zero terms corresponding to each $\widetilde{B}_{k}$ ).

The following useful characterization of which lacunary sequences lie in $A_{\alpha}^{p}$ may be known, but we include a proof for lack of a specific reference.

Proposition 2.1. Suppose $\left(a_{n}\right)$ is a lacunary sequence. Then, for any $0<p<\infty$, $\left(a_{n}\right) \in A_{\alpha}^{p}$ if and only if $\sum_{n=1}^{\infty} n^{-\alpha-1}\left|a_{n}\right|^{p}<\infty$.

Proof. The main idea is to write down the norm of a function as the sum of norms over the dyadic blocks, in the spirit of what is often called the Littlewood-Paley theory.

We may as well assume $\alpha=0$ by Corollary $1 \cdot 4(c)$. As a special case of $[\mathbf{6},(2 \cdot 3)$ and theorem $3 \cdot 1$ ], we have

$$
\|f\|_{A^{p}} \approx\left[\sum_{k=0}^{\infty} 2^{-k}\left\|w_{k} * f\right\|_{H^{p}}^{p}\right]^{1 / p},
$$


where, for each $k, w_{k}$ is a sequence whose terms are non-negative and bounded by 1 , and whose terms are zero for indices outside $\widetilde{B}_{k}$; additionally the sum over $k$ of the $n$th terms of the $w_{k} \mathrm{~s}$ is 1 for all $n$. If $p>1$ one can simply choose $w_{k}=\Delta_{k}$, the characteristic multiplier which is 1 on $B_{k}$ and zero elsewhere. For $p \leqslant 1$, it is shown in [25] that this simple but rough cutoff still gives one half of $(2 \cdot 2)$ (the right-hand side dominates the left) but to get the full-strength result, one must choose a smoother cutoff for large $k$.

The proposition therefore follows easily from the following two inequalities:

$$
\begin{aligned}
\left\|w_{k} * f\right\|_{H^{p}}^{p} & \leqslant C_{1} \sum_{n \in \widetilde{B}_{k}}\left|a_{n}\right|^{p}, \\
\sum_{j=k}^{k+1}\left\|w_{j} * f\right\|_{H^{p}}^{p} & \geqslant C_{2} \sum_{n \in B_{k}}\left|a_{n}\right|^{p},
\end{aligned}
$$

where $C_{1}$ and $C_{2}$ are independent of $k$. Since there are at most two non-zero terms with index in $\widetilde{B}_{k},(2 \cdot 3 a)$ is easily seen to be valid with $C_{1}=2 .(2 \cdot 3 b)$ is almost as easy to verify, so we leave it as an exercise with two hints: (1) if $n \in B_{k}$ then the $n$th coefficient of either $w_{k}$ or $w_{k+1}$ is at least $\frac{1}{2} ;(2)$ the $H^{p}$ norm of a power series remains unchanged if we divide by a power of $z$ to make the constant term non-zero.

Suppose $\left(\lambda_{n}\right)$ is lacunary and all of its terms are 0 or 1 . It is now obvious that $\left(\lambda_{n} n^{1 / p}\right)$ is in $A^{q}$ if and only if $0<q<p$. Also $\left(\lambda_{n} n^{1 / p} \log ^{-t} n\right) \in A^{p}$ if and only if $t p>1$. Considering the action of fractional differentiation on this last example shows that one cannot replace the inequality in Theorem $1 \cdot 3(b)$ or Corollary $1 \cdot 4(b)$ by equality.

As another application of Theorem 1·3, one can prove that Lemma 1.2(ii) is best possible in the sense of Duren-Taylor: if $\delta_{n}$ is any sequence tending to zero, $p \geqslant 1$, and $\alpha>-1$, then there are sequences $\left(a_{n}\right) \in A_{\alpha}^{p}$ such that $a_{n} \neq O\left(\delta_{n} n^{\frac{\alpha+1}{p}}\right)$. This was shown in [27] when $1 \leqslant p<2$ using lacunary sequences and the converse part of Lemma $1 \cdot 2$ (iv); but the argument works also for $p>2$ since Proposition $2 \cdot 1$ says that the converse part of Proposition $2 \cdot 1$ is also valid for $p>2$ if $\left(a_{n}\right)$ is lacunary (also the example given in [27] to prove that the exponent in Lemma $1 \cdot 2$ (ii) is best possible when $p>2$ does not work as claimed).

The second important class of examples we wish to examine are monotone sequences. For $p>1$, we characterize the monotonic sequences which are in $A^{p}$.

Proposition 2.4. Suppose $a_{n} \geqslant 0,1<p, C<\infty$ and either

(i) $\left(a_{n}\right)$ is monotonic, or

(ii) For each $k,\left(a_{n}\right)_{n \in B_{k}}$ is monotonic and, for all $n, m \in B_{k}, a_{m} \leqslant C_{o} a_{n}$.

Then $\left(a_{n}\right) \in A_{\alpha}^{p}$ if and only if $\sum_{n=1}^{\infty} n^{p-3-\alpha}\left|a_{n}\right|^{p}<\infty$. Furthermore, if $\left(a_{n}\right) \in A^{p}$, then $\left\|\left(a_{n}\right)\right\|_{A_{\alpha}^{p}}^{p} / \sum_{n=1}^{\infty} n^{p-3-\alpha}\left|a_{n}\right|^{p}$ is bounded above and below by constants dependent only on $p, \alpha$, and $C_{0}$.

Proof. As usual we may assume $\alpha=0$. We claim that for all $n \in B_{k}$, $\left\|\sum_{n \leqslant m \in B_{k}} z^{m}\right\|_{H^{p}} \leqslant C 2^{k(p-1) / p}$. One approach to justifying this is to write the sum as $\left(z^{2^{k}}-z^{n}\right) /(z-1)$ and find upper bounds for this expression for $z=e^{i \theta}$ in the ranges $|\theta| \leqslant 2^{-k}$ and $2^{-j-1}<|\theta| \leqslant 2^{-j}, j=0, \ldots, k$; in any case, we leave the details 
to the reader. Using monotonicity and the summation by parts formula, we have

$$
\sum_{n \in B_{k}} a_{n} z^{n}=a_{2^{k-1}} \sum_{n \in B_{k}} z^{n}+\sum_{2^{k-1}<n \in B_{k}}\left(a_{n}-a_{n-1}\right) \sum_{n \leqslant m \in B_{k}} z^{m}
$$

and therefore $\left\|\sum_{n \in B_{k}} a_{n} z^{n}\right\|_{H^{p}} \leqslant C 2^{k(p-1) / p}\left(a_{2^{k-1}}^{p}+a_{2^{k}-1}^{p}\right)^{1 / p}$.

As previously mentioned, $\|f\|_{A^{p}} \leqslant C\left[\sum_{k=0}^{\infty} 2^{-k}\left\|\Delta_{k} * f\right\|_{H^{p}}^{p}\right]^{1 / p}$ for all $p>0$, where $\Delta_{k}$ is the characteristic multiplier of $B_{k}$. Thus

$$
\|f\|_{A^{p}}^{p} \leqslant C\left[\sum_{k=0}^{\infty} 2^{k(p-2)}\left(a_{2^{k-1}}^{p}+a_{2^{k}-1}^{p}\right)\right]
$$

and one half of the equivalence follows.

Conversely, suppose $f \in A^{p}$. By Lemma 1.2(iii), it suffices to prove the desired implication for $p>2$. Using monotonicity and one direction of $(2 \cdot 2)$, the proof follows easily from the estimate $\left\|\sum_{n \in B_{k}} a_{n} z^{n}\right\|_{H^{p}} \geqslant C 2^{k(p-1) / p} \min _{n \in B_{k}} a_{n}$. This last inequality is immediate if one considers a lower bound on the real part of the left-hand sum on the arc $\left\{z=e^{i \theta}:|\theta|<2^{-k}\right\}$.

The final statement follows by an examination of the above proof, and of the proof of our Lemma 1.2(iii) given in [27].

\section{Self-multipliers}

A self-multiplier of $X$ is a member of $(X, X)$. Using Stirling's formula, we see that

$$
A \sum_{n=1}^{\infty}\left|a_{n}\right|^{2} / n^{1+\alpha} \leqslant\|f\|_{A_{\alpha}^{2}}^{2} \leqslant B \sum_{n=1}^{\infty}\left|a_{n}\right|^{2} / n^{1+\alpha},
$$

for some $0<A \leqslant B<\infty(A=B=1$ when $\alpha=0)$. Consequently, the selfmultipliers of $A_{\alpha}^{2}$ are precisely all bounded sequences. By Corollary $1 \cdot 4(d)$, we always have $\left(A_{\alpha}^{p}, A_{\alpha}^{p}\right)=\left(A^{p}, A^{p}\right)$, but no simple description of $\left(A^{p}, A^{p}\right)$ is known when $p \neq 2$. In this section, we discuss certain useful types of examples and non-examples of such self-multipliers.

We recall that a sequence space $X$ is said to be solid if $\left(b_{n}\right) \in X$ whenever $\left|b_{n}\right| \leqslant$ $\left|a_{n}\right|,\left(a_{n}\right) \in X$. The spaces $l^{p, q}$ and $c_{0}$ are examples of solid spaces. We shall see later that $\left(A^{p}, A^{p}\right)$ is not solid when $p \neq 2$ and so it cannot be expressed in terms of any of the standard (solid) sequence spaces.

Suppose $X^{p}=H^{p}$ or $A_{\alpha}^{p}, 0<p<\infty,-1<\alpha<\infty$. By considering vectors of the form $z^{n}$, the Closed Graph Theorem immediately implies that $\left(X^{p}, X^{p}\right) \subset l^{\infty}$ (and containment is strict whenever $p \neq 2$; see Corollary $4 \cdot 4$ ).

The shift operators $\sigma^{-}$and $\sigma^{+}$, as usual, are defined by

$$
\begin{aligned}
\sigma^{-}\left(a_{0}, a_{1}, a_{2}, \ldots\right) & =\left(a_{1}, a_{2}, \ldots\right), \\
\sigma^{+}\left(a_{0}, a_{1}, a_{2}, \ldots\right) & =\left(0, a_{0}, a_{1}, a_{2}, \ldots\right) .
\end{aligned}
$$

We call a topological vector space of analytic functions an $M$-space if it is metrizable by an invariant metric $d$ 'controlled by' $\left\{M_{p}(r, f): 1 / 2<r<1\right\}$ for some $p>0$, in the sense that if $M_{p}(r, f) \leqslant 2 M_{p}(r, g)$ for all $1 / 2<r<1$ then $d(f, 0) \leqslant C d(g, 0)$. For example, $H(p, q, s)$ is an $M$-space for any allowable $(p, q, s)$. We record here a simple lemma which we shall use implicitly wherever we need it. 
Lemma 3.1. $\sigma^{+}$and $\sigma^{-}$are bounded on all M-spaces.

Proof. Since $\sigma^{+}(f(z))=z f(z)$, we have $M_{p}\left(r, \sigma^{+}(f)\right) \leqslant M_{p}(r, f)$ for all $0<r<1$, and so $\sigma^{+}$is bounded. Similarly, $\sigma^{-}(f(z))=(f(z)-f(0)) / z$, and $M_{p}(r, f-f(0)) \leqslant$ $2 M_{p}(r, f)$ (since $f(0) \leqslant M_{p}(r, f)$ for all $p, r>0$ ). Thus $M_{p}\left(r, \sigma^{-}(f)\right) \leqslant 4 M_{p}(r, f)$ for any $\frac{1}{2}<r<1$, and so $\sigma^{-}$is bounded.

If $X$ and $Y$ are $M$-spaces, then $\lambda=\left(\lambda_{n}\right)_{n=0}^{\infty} \in(X, Y)$ if and only if $\left(\lambda_{n+1}\right)_{n=0}^{\infty} \in$ $(X, Y)$ (since the latter multiplier is simply $\left.\sigma^{-} \lambda \sigma^{+}\right)$. We shall use Lemma $3 \cdot 1$ mainly in this form as a convenience to simplify later statements. For example, $\left((n+1)^{t}\right)$ and $\left(n^{t}\right)$ are equivalent for all $t \in \mathbf{R}$.

We say that a vector space of analytic functions $X$ has the small multiplier property if there exists $r>0$ such that if $\lambda_{n}=O\left(n^{-r}\right)$ then $\lambda=\left(\lambda_{n}\right) \in(X, X)$. We say that a sequence $\left(\lambda_{n}\right)$ has Taylor form if there are sequences $\left(a_{i}\right),\left(C_{i}\right)$ such that,

$$
\forall k, n>0:\left|\lambda_{n}-\sum_{i=0}^{k} a_{i}(n+1)^{-i}\right| \leqslant C_{k}(n+1)^{-k-1} .
$$

We refer to the sequence $\left(p_{k, n}\right)_{n=1}^{\infty} \equiv\left(\sum_{i=1}^{k} a_{i}(n+1)^{-i}\right)$ as the $k$ th Taylor polynomial of $\lambda$ and $\left(\lambda_{n}-p_{k, n}\right)_{n=1}^{\infty}$ as the $k$ th remainder and $\left(a_{i}(n+1)^{-i}\right)_{n=1}^{\infty}$ as the $i$ th Taylor summand. Taylor form is a purely asymptotic property: it is invariant under changes of a finite number of terms. Also, the above type of expansion in negative powers of $n+1$ is equivalent to the corresponding expansion in negative powers of $n$ but is more convenient for the next lemma.

The following lemma is simple, but very useful - it is used (for example in Lemma $3 \cdot 8$, and $[8]$ to study compact multiplier operators) to prove that certain pairs of multipliers are equivalent on most function spaces when intuitively we expect this to be true.

Lemma 3·2. Suppose that $X$ is a vector space of analytic functions which has the small multiplier property, and that $\left((n+1)^{-1}\right) \in(X, X)$. Then all multipliers which have Taylor form are self-multipliers on $X$.

Proof. Let $\lambda$ have Taylor form. The $k$ th Taylor summand of $\lambda$ is a self-multiplier of $X$ for $k=0$ since $X$ is a vector space, and for all $k>0$ by iterating the fact that $\left((n+1)^{-1}\right) \in(X, X)$. By the small multiplier property, the $k$ th remainder of $\lambda$ is a self-multiplier of $X$ if $k$ is sufficiently large. It follows that $\lambda \in(X, X)$.

The hypotheses for $X$ in this lemma are quite weak. For example, we have the following lemma.

Lemma 3·3. All $H(p, q, s)$ spaces satisfy the hypotheses of Lemma $3 \cdot 2$.

Proof. To see that $X \equiv H(p, q, s)$ has the small multiplier property, we show that $\lambda \in\left(X, H^{\infty}\right)$ whenever $\lambda_{n}$ decreases at a sufficiently fast rate. By Lemma $1 \cdot 1, H^{p} \subset$ $H(p, q, t) \subset H(2,2, s)$ where $s=t+\max (0,1 / p-1 / 2)+\max (0,1 / 2-1 / q)$, and so it suffices to prove the result for $A_{\alpha}^{2}$, for all $\alpha>-1$. If $\left(a_{n}\right) \in A_{\alpha}^{2}$ then $a_{n}=O\left(n^{(1+\alpha) / 2}\right)$, and so $\lambda \in\left(A_{\alpha}^{2}, H^{\infty}\right)$ if $\lambda_{n}=O\left(n^{-2-\alpha / 2}\right)$, say.

The fact that $\left((n+1)^{-1}\right) \in(X, X)$ follows from Lemma 1.1(iv) in all cases except $X=H^{p}$. In this case, it is true by a theorem of Hardy and Littlewood $[10$, theorem $5 \cdot 12]$. 
Fractional integration, differentiation, and weighted Bergman spaces 377

Let $B(\cdot, \cdot)$ be the classical beta function. The alternative fractional integration and differentiation multipliers $\tilde{I}^{t}=\left((B(n+1, t))\right.$ and $\tilde{D}^{t}=(1 / B(n+1, t))$, are used by some authors. We shall use Lemma $3 \cdot 2$ to prove that the two types of fractional operations are equivalent on $H(p, q, s)$. This equivalence was proved in certain cases by Flett [15, theorem 7]. Here we show that if $X$ is any space of analytic functions satisfying the hypotheses of Lemma $3 \cdot 2$ then these operators are equivalent on $X$.

Lemma 3·4. If $\lambda \equiv\left(\lambda_{n}\right)=\left(n^{t} B(n+1, t)\right)_{n=1}^{\infty}$ for some $t>0$, then both $\lambda$ and $\lambda^{-1} \equiv$ $\left(1 / \lambda_{n}\right)$ have Taylor form.

Proof. We recall the following asymptotic expansion of $\Gamma(x+1)$ (see, for instance, $[24$, p. 380]):

$$
\Gamma(x+1)=\sqrt{2 \pi} x^{x+\frac{1}{2}} e^{-x}\left(1+\frac{1}{12 x}+\cdots+\frac{a_{k}}{x^{k}}+O\left(x^{-k-1}\right)\right) .
$$

Thus

$$
\frac{n^{t} B(n+1, t)}{\Gamma(t)}=e^{t}\left(\frac{n}{n+t}\right)^{n+t+\frac{1}{2}} \frac{1+(1 / 12 n)+\cdots+\left(a_{k} / n^{k}\right)+O\left(n^{-k-1}\right)}{1+(1 / 12(n+t))+\cdots+\left(a_{k} /(n+t)^{k}\right)+O\left(n^{-k-1}\right)} .
$$

Thus, to show that $\lambda$ has Taylor form, it suffices to show that the middle factor has Taylor form. Since

$$
x \mapsto(1+t x)^{-t-1 / 2-1 / x}=e^{-\log (1+t x)(t+1 / 2+1 / x)}
$$

has a Taylor series expansion about zero (with radius of convergence $1 / t$ ), the required result follows by letting $x=1 / n$. Finally, it is clear from the above that $\lambda^{-1}$ also has Taylor form.

Corollary 3.5. Suppose $X$ is a vector space of analytic functions satisfying the assumptions of Lemma $3 \cdot 2$, and that $f$ is an analytic function. Then for any $t>0$, $I^{t} f \in X$ if and only if $\tilde{I}^{t} f \in X$, and $D^{t} f \in X$ if and only if $\tilde{D}^{t} f \in X$.

We denote by $B V$ the classical space of (complex) sequences of bounded variation:

$$
B V=\left\{\left(\lambda_{n}\right)_{n=0}^{\infty}:\left\|\left(\lambda_{n}\right)\right\|_{B V}=\left|\lambda_{0}\right|+\sum_{n=0}^{\infty}\left|\lambda_{n+1}-\lambda_{n}\right|<\infty\right\} .
$$

As is also well known, a sequence belongs to $B V$ if and only if both its real and imaginary parts can be written as differences of two bounded monotonic sequences. Note that $B V$ is not solid and that all Taylor form multipliers have bounded variation (but there are many $B V$ sequences, such as $\left((-1)^{n} /(n+1)^{2}\right)$, which do not have Taylor form).

Unlike Taylor form multipliers, $B V$ sequences are not necessarily self-multipliers of $A^{p}$. In fact, we shall see that $B V \subset\left(A^{p}, A^{p}\right)$ if and only if $p>1$. Let us first prove that $B V \subset(X, X)$ when $X$ is any one of many Banach spaces of analytic functions. For this we need a lemma which is due (at least partially) to Zhu [32].

Lemma 3.6. For $X=A^{p}$ or $H^{p}, 1<p<\infty$ and $f \in X$ the sequence $\left(P_{n} f\right)_{n=0}^{\infty}$ of Taylor polynomials converges to $f$ in the norm of $X$. The statement is false for $p=1$.

Proposition 3.7. Let $(X,\|\cdot\|)$ be a Banach space of analytic functions in the unit disc $\mathbf{D}$ such that for every $f \in X$ the sequence $\left(P_{n} f\right)_{n=1}^{\infty}$ of Taylor polynomials converges 
to $f$ in the norm of $X$. Then $B V \subset(X, X)$, and the inclusion is strict if the involution $T f(z)=f(-z)$ is bounded on $X$. In particular, $B V$ is a proper subset of $(X, X)$ if $X=A^{p}$ or $H^{p}, 1<p<\infty$.

Proof. Let $f(z)=\sum_{k=0}^{\infty} a_{k} z^{k} \in X$, and choose $M>0$ such that $\left\|\left(\lambda_{n}\right)\right\|_{B V} \leqslant M$. Given $\epsilon>0$, we have for sufficiently large $n$ that

$$
\left\|\sum_{k=n}^{\infty} a_{k} z^{k}\right\|<\frac{\epsilon}{2 M}
$$

(such a choice is possible by our assumption on $P_{n} f$ ). A summation by parts yields $\left\|\sum_{k=n}^{\infty} \lambda_{k} a_{k} z^{k}\right\|=\left\|\sum_{k=n+1}^{\infty}\left(\lambda_{k+1}-\lambda_{k}\right) \cdot \sum_{j=k}^{\infty} a_{j} z^{j}-\lambda_{n} \sum_{k=n}^{\infty} a_{k} z^{k}\right\|<M \frac{\epsilon}{2 M}+M \frac{\epsilon}{2 M}=\epsilon$,

for all sufficiently large $n$. Thus, $\sum_{k=0}^{\infty} \lambda_{k} a_{k} z^{k} \in X$, which proves the claim. In the case $X=A^{p}$ or $H^{p}, 1<p<\infty$, the statement follows from Lemma $3 \cdot 6$.

The involution $T$ corresponds to the sequence $\left((-1)^{n}\right)$ which is not in $B V$, and so the inclusion is strict if $T \in(X, X)$. Obviously, $T$ is bounded on all $H(p, q, s)$ spaces, including $A^{p}$ and $H^{p}, p>0$.

We now define the one-parameter family $f_{t}(z)=(1-z)^{-t}$, for all $t>0$. Simple calculations show that $f_{t} \in A^{p}$ if and only if $t p<2$, and $f_{t} \in H^{p}$ if and only if $t p<1$. Since the $n$th coefficient of $f_{t}$ is $1 / n B(n, t)$, our results on Taylor form multipliers tell us that $f_{t} \in A^{p}$ if and only if $D^{t-1} \in A^{p}$ (i.e. if and only if $\sum_{n=1}^{\infty} n^{t-1} z^{n} \in A^{p}$ ), and similarly for $H^{p}$.

Lemma 3.8. $D^{t} \in A^{p}$ if and only if $(t+1) p<2$, and $D^{t} \in H^{p}$ if and only if $(t+1) p<1$.

Proof. The lemma is trivially true if $t<-1$, and the preceding comments prove the case $t>-1$. Finally $D^{-1}=(1-z)[\log (1-z)-1]$, which clearly lies in $H^{\infty}$.

Using $D^{s}$ as a counterexample is essentially equivalent to using $f_{s+1}$, but the use of $D^{s}$ minimises the need to mention Taylor form multipliers. As a first application, it is now clear that the bounds on $t$ in Theorem $1 \cdot 3(a)$ and Corollary 1.4( $a)$ are best possible.

According to Proposition $3 \cdot 7$ every sequence of bounded variation is in $\left(A^{p}, A^{p}\right)$ for all $p>1$. Our next theorem tells us that this is false if $p \leqslant 1$. The proof gives two different methods of constructing counterexamples: the first is simpler but works only if $p<1$, while our $p=1$ method is easily adapted to handle all $p \leqslant 1$, but is less explicit and involves more background work. The case $p<\frac{2}{3}$ can also be proved from Theorem $4 \cdot 5$ below, a task we leave to the reader.

Theorem 3.9. For all $0<p \leqslant 1$ there exist monotonic $c_{0}$ sequences not in $\left(A^{p}, A^{p}\right)$.

Proof. Assume first that $p<1$. The sequence $\left(n^{s}\right)$ is in $A^{p}$ for all $s<2 / p-1$ but, if we perturb its $2^{k}$ th coefficient for all $k>1$ by subtracting $2^{k / p}$, the resulting sequence is not in $A^{p}$ by Proposition $2 \cdot 1$. Consequently, $\lambda=\left(\lambda_{n}\right) \notin\left(A^{p}, A^{p}\right)$ if

$$
\lambda_{n}= \begin{cases}1-2^{k(1 / p-s)}, & n=2^{k}, k>1 \\ 1 & \text { otherwise. }\end{cases}
$$


Thus if we choose any $s$ in the interval $(1 / p, 2 / p-1)$, the resulting $\lambda$ is a nonmultiplier of bounded variation. A monotonic decreasing $c_{0}$ non-multiplier is then easily found. One example is the function $f=\left(\mu_{n}\right)$ where

$$
\mu_{n}= \begin{cases}\sum_{j=1}^{\infty} 2^{j(1 / p-s)}, & \text { if } n<2, \\ \sum_{j=k+1}^{\infty} 2^{j(1 / p-s)}, & \text { if } n \in B_{k}, k>1 .\end{cases}
$$

To see this, note that the $n$th Taylor coefficient of $1+f(z)-z f(z)$ is $\lambda_{n}$.

This leaves the case $p=1$. It suffices to find a bounded variation non-multiplier (since such a sequence can be written as a difference of two monotonic $c_{0}$ sequences, at least one of which must be a non-multiplier). Suppose that no such sequence exists and so, by the Closed Graph Theorem, all $B V$ sequences are bounded as (multiplier) operators on $A^{1}$.

We shall need a slight variant of the characterization of $A^{1}$ given by the $p=1$ case of $(2 \cdot 2)$. As given earlier, the functions $w_{k}$ have non-zero terms only for indices in the range $2^{k-2}$ to $2^{k}$. This is because the $n$th term of $w_{k}$, which we denote by $w_{k, n}$ equals $\phi\left(n / 2^{k-2}\right)$, where $\phi(t)=\omega(t / 2)-\omega(t)$ and $\omega$ is any infinitely differentiable function defined on the interval $[0, \infty)$ satisfying $\omega(t)=1$ if $t \leqslant 1, \omega(t)=0$ if $t \geqslant 2$, and $0 \leqslant \omega(t) \leqslant 1$ if $1 \leqslant t \leqslant 2$. If we widen the allowable class of functions to include all smooth $\omega$ which are 1 for $t \leqslant 1,0$ for $t \geqslant 4$, and take on intermediate values on the interval [1,4], (2.2) is still valid since the proof in [19] of the crucial inequality $\left\|w_{k} * f\right\|_{H^{1}} \leqslant\|f\|_{H^{1}}$, which is needed in [6] to prove (2.2), goes through essentially unchanged (note that our $w_{k}$ corresponds to $w_{k-1}$ in the other two papers).

Suppose therefore that we choose such an $\omega$ so that it is strictly increasing on the interval $[1,4]$. It follows that the resulting function $\phi$ has a positive minimum on the interval [1,2] and hence that there exists $0<c<1$ such that for all $k>1$ and all $n \in B_{k}$, the $n$th term of $w_{k}$ lies between $c$ and 1 . Also, it is easy to show (see [25] for example) that if the Taylor coefficients of $f$ are zero outside the range from $m$ to $n$ then $M_{p}(r, f) /\|f\|_{H^{p}}$ lies between $r^{n}$ and $r^{m}$; hence it is true in general that $\left\|w_{k} * f\right\|_{H^{1}} \approx 2^{k}\left\|w_{k} * f\right\|_{A^{1}}$.

By (2.2), we have $\left\|w_{k} * f\right\|_{H^{1}} \leqslant C 2^{k}\|f\|_{A^{1}}$ (for some constant $C$ independent of $k$ ). On the other hand, functions $f_{k}$ are given in [25] for all $k>0$ such that all their Taylor coefficients with index in $B_{k}$ are positive, and $\left\|\Delta_{k} f_{k}\right\|_{H^{1}} \geqslant C k 2^{k}\|f\|_{A^{1}}$. Letting $\lambda_{k}=\left(\lambda_{k, j}\right)_{j=0}^{\infty}$ be defined by $\lambda_{k, j}=1 / w_{k, j}$, if $j \in B_{k}$, and $\lambda_{k, j}=0$ otherwise, we see that $\lambda_{k}\left(g_{k}\right)=\Delta_{k} f_{k}$, where we define $g_{k}=w_{k} * f_{k}$. By construction, $\left\|\lambda_{k}\right\|_{B V} \leqslant T+2 / c$, where $T$ is the total variation of the function $1 / \phi$ on $[1,2]$; in particular, the total variation of $\lambda_{k}$ has a bound independent of $k$. Writing $\lambda=\sum_{k=1}^{\infty} k^{-2} \lambda_{k^{3}}$, it follows that $\lambda \in B V$, but $\left\|\lambda\left(g_{k^{3}}\right)\right\|_{A^{1}} \geqslant C k\left\|g_{k^{3}}\right\|_{A^{1}}$, giving the required contradiction.

Our $B V$-related results can be summarized as follows.

Theовем 3.10. Each of the following statements about $A^{p}$ is true if and only if $p>1$.

(a) $B V \subset\left(A^{p}, A^{p}\right)$.

(b) Every monotone (real) $c_{0}$ sequence is a self-multiplier of $A^{p}$.

(c) If $f \in A^{p}$, the sequence $\left(P_{n} f\right)_{n=0}^{\infty}$ of Taylor polynomials is norm-convergent to $f$.

\section{Sequential estimates for Bergman space multipliers}

In this section, we investigate $\left(A_{\alpha}^{p}, A_{\beta}^{q}\right)$. The first statement gives a complete characterisation in the case $0<q \leqslant 2 \leqslant p<\infty$. The unweighted version $(\alpha=\beta=0)$ 
of this is due to Wojtaszczyk [30]. The weighted version given here is due to Anderson [2], who gave a considerably simpler and more elementary proof (of course in light of Corollary 1.4, the unweighted result immediately gives the weighted result). Elementary proofs in general situations were also given in [6] and [18]. Here and subsequently, $0^{-1}$ is defined to be $\infty$.

Lemma 4·1. Suppose $0<q \leqslant 2 \leqslant p<\infty$, and $\alpha, \beta>-1$. Then $\lambda=\left(\lambda_{n}\right) \in\left(A_{\alpha}^{p}, A_{\beta}^{q}\right)$ if and only if $\left(n^{-r} \lambda_{n}\right) \in l\left(\infty, s^{-1}\right)$, where $s=q^{-1}-p^{-1}$ and $r=(1+\beta) q^{-1}-(1+\alpha) p^{-1}$.

Lemma $4 \cdot 1$ tells us that $\left(A^{p}, A^{q}\right)$ is solid if $p \geqslant 2 \geqslant q$. The next two theorems, which deal with fractional differentiation composed with a random change of sign, will imply that this is false (for Hardy as well as Bergman spaces) if $p, q$ are either both less than 2 or both greater than 2 .

Theorem 4.2. Suppose that either $0<p, q \leqslant 2, p \neq 2$, or $2 \leqslant p, q<\infty, q \neq 2$. In the first case, we define $t_{0}=1 / q+1 / 2-2 / p$, and in the second case we define $t_{0}=$ $2 / q-1 / 2-1 / p$. If $t>t_{0}$, then for almost every choice of signs $\epsilon_{n}= \pm 1,\left(\epsilon_{n} n^{t}\right) \notin\left(A^{p}, A^{q}\right)$.

Proof. Consider first the case $p<2, q \leqslant 2$. Let $0<\delta \leqslant 1 / p-1 / 2$ be fixed but otherwise arbitrary. Lemma 3.8 implies that $D^{2 / p-1-\delta} \in A^{p} \backslash A^{2}$. By Lemma 1.1(v) and the upper bound on $\delta$, we deduce that $D^{1 / p-1-\delta} \in H^{p} \backslash H^{2}$.

If $\left(\epsilon_{n} n^{t}\right) \in\left(A^{p}, A^{q}\right)$ for $t=1 / q+1 / 2-2 / p+\delta$, one immediately obtains $\left(\epsilon_{n} n^{1 / q-1 / 2}\right)$ $\in A^{q}$. Using Lemma $1 \cdot 1(\mathrm{v})$ again, we see that $\left(\epsilon_{n} n^{-\frac{1}{2}}\right) \in H^{q}$. Since $\left(n^{-\frac{1}{2}}\right) \notin H^{2}$, a theorem of Littlewood [10, theorem A5] tells us that for almost every choice of signs $\epsilon_{n}= \pm 1,\left(\epsilon_{n} n^{-\frac{1}{2}}\right)$ has radial limit almost nowhere (and hence cannot lie in $H^{s}$ for any $s>0)$.

Since $\left(A^{p}, A^{q}\right)=\left(A^{q^{\prime}}, A^{p^{\prime}}\right)$ where $p^{\prime}, q^{\prime}$ are conjugate to $p, q$, the case $p \geqslant 2, q>2$ follows by duality.

Theorem $4 \cdot 3$. Suppose that $0<p, q<\infty$. Let $t_{0}=\min \{1 / 2-1 / p, 1 / q-1 / 2,0\}$. If $t>t_{0}$, then for almost every choice of signs $\epsilon_{n},\left(\epsilon_{n} n^{t}\right) \notin\left(H^{p}, H^{q}\right)$.

Proof. Fix $\delta>0$. By Lemma $3 \cdot 8, D^{1 / p-1-\delta} \in H^{p}$ and so it follows as in Theorem $4 \cdot 2$ that $\left(\epsilon_{n} n^{\delta+1 / 2-1 / p}\right) \notin\left(H^{p}, H^{q}\right)$ for almost every $\left(\epsilon_{n}\right)$. If $p, q>1$ then, by duality, $\left(\epsilon_{n} n^{\delta+1 / q-1 / 2}\right) \notin\left(H^{p}, H^{q}\right)$ for almost every $\left(\epsilon_{n}\right)$ (and note that $1 / q-1 / 2<$ $\min \{1 / 2-1 / p, 0\}$ forces $p, q>1)$. Finally, to see that $\left(\epsilon_{n} n^{\delta}\right) \notin\left(H^{p}, H^{q}\right)$, we note that $\left(n^{-\frac{1}{2}-\delta / 2}\right) \in H^{2}$ and so $\left(\epsilon_{n}^{\prime} n^{-\frac{1}{2}-\delta / 2}\right) \in H^{p}$ for some fixed choice of signs $\left(\epsilon_{n}^{\prime}\right)$, but $\left(n^{-\frac{1}{2}+\delta / 2}\right) \notin H^{2}$ and so $\left(\epsilon_{n} \epsilon_{n}^{\prime} n^{-\frac{1}{2}+\delta / 2}\right) \notin H^{p}$ for almost all $\left(\epsilon_{n}\right)$.

Corollary 4.4. If $0<p, q<2$ or $2<p, q<\infty$ then $\left(A^{p}, A^{q}\right)$ and $\left(H^{p}, H^{q}\right)$ are non-solid.

Proof. It is an easy consequence of Theorem 1.3 that $D^{t} \in\left(A^{p}, A^{q}\right)$ for all $t>t_{0}$ whenever $p, q, t_{0}$ are as in Theorem $4 \cdot 2$. Thus, $\left(A^{p}, A^{q}\right)$ is not solid if $p, q$ are as in the hypothesis.

Letting $t_{1}=0$ if $q \leqslant p$ and $t_{1}=1 / q-1 / p$ if $q>p$, we have $D^{t_{1}} \in\left(H^{p}, H^{q}\right)$ (this is obvious if $p \leqslant q$, while the $p>q$ case is the Hardy-Littlewood theorem on fractional integrals; see, for example [14, theorem 9]). If $p, q$ are as hypothesized, it is readily verified that $t_{1}$ is larger than the index $t_{0}$ of Theorem $4 \cdot 3$, and so $\left(H^{p}, H^{q}\right)$ is not solid. 
Fractional integration, differentiation, and weighted Bergman spaces 381

For $0<p<2<q<\infty$, it was proved by the third author [29, theorem 2] that $\lambda=\left(\lambda_{n}\right) \in\left(A^{p}, A^{q}\right)$ if $\lambda_{n}=O\left(n^{2 / q-2 / p}\right)$, and that this index is best possible. We now recover this result by applying Corollary 1.4 (or Theorem $1 \cdot 3$ ) to Lemma $4 \cdot 1$. More generally, we shall get separate necessary and sufficient conditions for a sequence to be in $\left(A_{\alpha}^{p}, A_{\beta}^{q}\right)$ for all allowable values of $p, q, \alpha, \beta$. We begin with the sufficient conditions.

Theorem 4.5. Suppose $p, q>0$, and $\alpha, \beta>-1$. Let $p_{1}=\max \{p, 2\}, q_{1}=\min \{q, 2\}$, $s=q_{1}^{-1}-p_{1}^{-1}$, and $r=(2+\beta) q^{-1}-(2+\alpha) p^{-1}-s$. If

$$
\left(n^{-r} \lambda_{n}\right) \in l\left(\infty, s^{-1}\right)
$$

then $\lambda=\left(\lambda_{n}\right) \in\left(A_{\alpha}^{p}, A_{\beta}^{q}\right)$. The index $r$ is best possible in all cases.

Proof. The case $0<q \leqslant 2 \leqslant p<\infty$ is one direction of Lemma 4.1. We look at the case $0<p<2<q<\infty$. By Theorem 1.3, $I^{t_{1}} \in\left(A_{\alpha}^{p}, A_{\alpha}^{2}\right)$ and $I^{t_{2}} \in\left(A_{\beta}^{2}, A_{\beta}^{q}\right)$, where $t_{1}=(2+\alpha)\left(p^{-1}-2^{-1}\right)$ and $t_{2}=(2+\beta)\left(2^{-1}-q^{-1}\right)$. By a (trivial) special case of Lemma $4 \cdot 1, \mu_{n} \in\left(A_{\alpha}^{2}, A_{\beta}^{2}\right)$ if and only if $\left(n^{(\alpha-\beta) / 2} \mu_{n}\right) \in l^{\infty}$. Putting everything together, we get that $\left(\lambda_{n}\right)=\left(n^{-t_{1}-t_{2}} \mu_{n}\right) \in\left(A_{\alpha}^{p}, A_{\beta}^{q}\right)$ if $n^{t_{1}+t_{2}+(\alpha-\beta) / 2} \lambda_{n}$ is bounded. Since

$$
t_{1}+t_{2}+(\alpha-\beta) / 2=(2+\alpha) p^{-1}-(2+\beta) q^{-1}
$$

and $s=0$, we obtain $(4 \cdot 6)$.

In the case $0<p, q<2, \lambda \in\left(A_{\alpha}^{p}, A_{\beta}^{q}\right)$ if $\lambda_{n}$ can be written as $n^{-t_{1}} \mu_{n}$, where $t_{1}$ is as before, and $\left(\mu_{n}\right) \in\left(A_{\alpha}^{2}, A_{\beta}^{q}\right)$; in the case $p, q>2, \lambda \in\left(A_{\alpha}^{p}, A_{\beta}^{q}\right)$ if $\lambda_{n}$ can be written as $n^{-t_{2}} \mu_{n}$, where $t_{2}$ is as before, and $\left(\mu_{n}\right) \in\left(A_{\alpha}^{p}, A_{\beta}^{2}\right)$. In both cases, (4.6) follows by applying Lemma $4 \cdot 1$ to $\left(\mu_{n}\right)$.

It remains to show that $r$ in $(4 \cdot 6)$ is maximal. By Corollary $1 \cdot 4$, we may assume without loss of generality that $\alpha=\beta=0$. By an easy dyadic-block argument, it is equivalent to show that for every $\delta>0$, there are sequences $\left(\lambda_{n}\right)=O\left(n^{r+\delta}\right)$ not in $\left(A^{p}, A^{q}\right)$. For the case $0<p<2<q<\infty$, it suffices to note that if $\delta>0$ then $D^{-\delta+2 / p-1} \in A^{p}$, but $D^{r} D^{-\delta+2 / p-1} \notin A^{q}$ if $r \geqslant \delta+2 / q-2 / p$. Sharpness for $p<2, q \leqslant 2$ and for $p>2, q \geqslant 2$ follows from Theorem $4 \cdot 2$ (and conversely the sharpness of the index $t_{0}$ in Theorem $4 \cdot 2$ follows from this theorem). The case $p \geqslant 2 \geqslant q$ follows from Lemma $4 \cdot 1$.

We now state the corresponding necessary conditions for a sequence to be a multiplier (Theorems $4 \cdot 7$ and $4 \cdot 8$ ). A weaker version of Theorem 4.7 could be proved in a similar fashion to Theorem $4 \cdot 5$ (with fractional differentiation replacing fractional integration), but lacunary sequences provide a simpler route to the full-strength result. Note that the exponent $r$ in Theorem 4.7 is sharp when $p \geqslant 1$, but not when $p<1$. In the latter case, Theorem 4.8 gives the sharp order of growth.

Theorem 4.7. Suppose $p, q>0, \alpha, \beta>-1, r=(1+\beta) q^{-1}-(1+\alpha) p^{-1}$, and $s=\max \left\{0, q^{-1}-p^{-1}\right\}$. If $\lambda=\left(\lambda_{n}\right) \in\left(A_{\alpha}^{p}, A_{\beta}^{q}\right)$ then $\left(n^{-r} \lambda_{n}\right) \in l\left(\infty, s^{-1}\right)$. The converse is true if $p \geqslant 1$ and $\lambda$ is lacunary.

Proof. By Corollary 1.4, we may as well assume $\alpha=\beta=0$. Let $\left(a_{k}\right)$ be a lacunary sequence whose terms are zero except possibly at positions $n_{k} \in B_{k}, k \geqslant 0$. Writing $b_{k}=2^{-k / p} a_{n_{k}}$, the assumption $\left(a_{n}\right) \in A^{p}$ is equivalent to $\left(b_{k}\right) \in l^{p}$. Similarly applying 
$\lambda \in\left(A^{p}, A^{q}\right)$ to $\left(a_{n}\right)$, we get $\left(b_{k} \lambda_{n_{k}} 2^{k(1 / p-1 / q)}\right) \in l^{q}$. Since $n_{k} \in B_{k}$ is arbitrary, it follows that $\left(b_{k} \mu_{k}\right) \in l^{q}$ for every $\left(b_{k}\right) \in l^{p}$, where $\mu_{k}=2^{k(1 / p-1 / q)} \max \left\{\left|\lambda_{n}\right|: n \in B_{k}\right\}$. The required necessity now follows from the well-known result that $\left(l^{p}, l^{q}\right)=l^{1 / s}$.

For the partial converse, suppose $\lambda$ is lacunary and $\left(n^{-r} \lambda_{n}\right) \in l\left(\infty, s^{-1}\right)$. Thus $\lambda_{n}=0$ unless $n=n_{k} \in B_{k}$ and $\left(n_{k}^{-r} \lambda_{n_{k}}\right) \in l^{1 / s}$. The Cauchy integral formula implies that if $f=\left(b_{n}\right) \in H^{p}$, then $\left|b_{n}\right| \leqslant\|f\|_{H^{p}}$ for all $p \geqslant 1$. This and (2.2) imply that if $\left(a_{n}\right) \in A^{p}$ then $\left(n_{k}^{-1 / p} a_{n_{k}}\right) \in l^{p}$. Since $\left(l^{p}, l^{q}\right)=l^{1 / s}$, the required result follows.

Theorem 4.8. Suppose $0<p<1,0<q<\infty$ and $\alpha, \beta>-1$. Let

$$
r= \begin{cases}\frac{1+\beta}{q}-\frac{2+\alpha}{p}+1, & \text { if } 1 \leqslant q \\ \frac{2+\beta}{q}-\frac{2+\alpha}{p}, & \text { if } p<q<1 \\ \frac{1+\beta}{q}-\frac{1+\alpha}{p}, & \text { if } q \leqslant p .\end{cases}
$$

If $\lambda=\left(\lambda_{n}\right) \in\left(A_{\alpha}^{p}, A_{\beta}^{q}\right)$ then $\lambda_{n}=O\left(n^{r}\right)$. Furthermore, given any values of $p, q, \alpha, \beta$, the number $r$ is minimal in the last statement.

Proof. As usual, we may assume $\alpha=\beta=0$. Theorem $4 \cdot 7$ implies (a sharper version of) the case $q \leqslant p$, so we need only look at the other two cases. By Lemma $3 \cdot 8$, $\lambda D^{s} \in A^{q}$ for each $s<2 / p-1, \lambda \in\left(A^{p}, A^{q}\right)$, and so by parts (i) and (ii) of Lemma 1.2, we must have $\lambda_{n}=O\left(n^{t}\right)$ for any $t>r$. This is close to what we need but we need to argue a little more delicately to get $t=r$.

We first note that $\left(A^{p}, l^{\infty}\right)=\left\{\left(\lambda_{n}\right): n^{2 / p-1} \lambda_{n} \in l^{\infty}\right\}$, whenever $0<p \leqslant 1$. This is a special case of a result of Blasco $[6,4 \cdot 5]$ (alternatively note that containment in one direction follows from Lemma $1 \cdot 2(\mathrm{i})$, and the opposite direction follows from the fact that $H^{p / 2} \subset A^{p}$ and the corresponding result for $H^{p}$ in $[\mathbf{1 0}$, theorem $\left.\mathbf{6 \cdot 5}]\right)$. It follows that if $0<1 / d_{n} \neq O\left(n^{1-2 / p}\right)$ then there exists $\left(a_{n}\right) \in A^{p}$ such that $a_{n} \neq O\left(d_{n}\right)$ (otherwise $\left.\left(1 / d_{n}\right) \in\left(A^{p}, l^{\infty}\right)\right)$. Suppose therefore that $\lambda_{n} \neq O\left(n^{r}\right)$ and so there exists a sequence of integers $n_{k}$ such that $\lambda_{n_{k}}>k^{2} n_{k}^{r}$. Choosing $\left(a_{n}\right) \in A^{p}$ such that $k\left|a_{n_{k}}\right|>n_{k}^{2 / p-1}$, we see that $\left(\lambda_{n} a_{n}\right)$ cannot be in $A^{q}$ since it violates Lemma $1 \cdot 2(\mathrm{i})$ $(q<1)$ or Lemma $1 \cdot 2($ ii) $)(1 \leqslant q)$.

We are left with proving minimality of $r$. In the case $q \geqslant 1$, simply consider lacunary multipliers and use Lemma 1.2(i). Parts $(a)$ and $(b)$ of Corollary 1.4 imply $r$ is minimal when $0<p \leqslant q<1$ and when $0<q<p<1$, respectively.

We now compare our $L^{\infty}$-type necessary/sufficient multiplier estimates with the $L^{q}$-type estimates for multipliers between unweighted Bergman spaces of [29]. Our sufficient conditions are easily seen to imply the following result.

Corollary 4.9. In any of the following circumstances, $\lambda=\left(\lambda_{n}\right) \in\left(A^{p}, A^{q}\right)$.

(i) $0<p, q \leqslant 2$ and $\sum_{n=1}^{\infty} n^{-1-q / 2+2 q / p}\left|\lambda_{n}\right|^{q}<\infty$.

(ii) $p, q \geqslant 2$ and $\sum_{n=1}^{\infty} n^{-2+q / 2+q / p}\left|\lambda_{n}\right|^{q}<\infty$.

(iii) $p \leqslant 2 \leqslant q$ and $\sum_{n=1}^{\infty} n^{-2+2 q / p}\left|\lambda_{n}\right|^{q}<\infty$.

(iv) $q \leqslant 2 \leqslant p$ and $\sum_{n=1}^{\infty} n^{-1+q / p}\left|\lambda_{n}\right|^{q}<\infty$.

Proposition 1 of [29] also gives a sufficient condition for each of four cases, but the powers of $n$ in the sums are different and they change at $p=1$ rather than $p=2$ (see 
Fractional integration, differentiation, and weighted Bergman spaces 383

[29] for details). Naturally, one can also use the $L^{q}$ estimates of [29] to derive $L^{\infty}$ estimates. Doing so, one sees that the two sets of results are incomparable if $0<p \leqslant$ $1, q>0$ and also if $1<p<2, q<p^{\prime}$. However, Theorem 4.5 implies Proposition 1 when $1<p \leqslant 2 \leqslant p^{\prime}<q$ and when $p>2, q>0$; in fact if $q>\max \left(2, p^{\prime}\right)$ then the condition in Corollary 4.9 has a strictly smaller power of $n$ than its counterpart in Proposition 1.

We next examine $L^{q}$ necessary conditions to be a multiplier. Since it is routine to derive such conditions from Theorems $4 \cdot 7$ and $4 \cdot 8$, we state a result only for the case where we get better estimates than those of the corresponding theorem 8 of [29]; we use ' $O(N)$ ' format for greater ease of comparability with theorem 8.

Corollary 4.10. Suppose $\lambda=\left(\lambda_{n}\right) \in\left(A^{p}, A^{q}\right)$ and either $0<q \leqslant p$ or $p \geqslant 1, q>0$. Then $\sum_{n=1}^{N} n^{q / p-1-\epsilon}\left|\lambda_{n}\right|^{q}=O(N)$ for every $\epsilon>0$.

Corollary $4 \cdot 10$ is stronger than Theorem 8 when $q<\min (2, p)$ and also when $1<p<2, q>p^{\prime}$. In all other cases where it is applicable it is weaker; in fact, Theorems 8 and $4 \cdot 7,4 \cdot 8$ are incomparable. In cases not handled by Corollary $4 \cdot 10$, Theorems 8 and 4.8 are also incomparable except when $0<p<1, q \geqslant 2$. Theorem 8 states that if $\lambda \in\left(A^{p}, A^{q}\right)$ for some $0<p<\infty, 2 \leqslant q<\infty$, then $\sum_{n=1}^{N} n^{2 q / p-q}\left|\lambda_{n}\right|^{q}=O(N)$. This implies that $\left(A^{p}, A^{q}\right)$ multipliers $(q \geqslant 2)$ have terms which are $o\left(n^{1 / q^{+1-2 / p}}\right)$, a sharper version of Theorem $4 \cdot 8$ for $0<p<1, q \geqslant 2$.

\section{Univalent Bergman functions}

The integrability of derivatives of conformal maps is one of the central questions in function theory. We show here (at least in the unweighted case) that the gap in Corollary 1.4 between fractional integration and differentiation (parts $(a)$ and $(b)$ ) can be bridged 'to within $\epsilon$ ' if the function is univalent. Below, $\mathscr{U}$ is the class of functions univalent on $\mathbf{D}$, and $S \subset \mathscr{U}$ denotes the normalised univalent functions that satisfy $f(0)=0$ and $f^{\prime}(0)=1$.

Proposition 5·1. If $f \in A^{p} \bigcap \mathscr{U}$ and $0<t \leqslant 1$ then $D^{t} f \in A^{q}$ for all $q^{-1}>p^{-1}+t / 2$.

Proof. It was shown in $[\mathbf{2 0}]$ that if a quasiconformal mapping on the unit ball $B \subset \mathbf{R}^{n}$ is in $L^{p}(B)$, then its derivative is in $L^{q}(B)$ for all $q^{-1}>p^{-1}+1 / n$. Since univalent mappings are quasiconformal, the $n=2$ case gives the $t=1$ case of our result. The case $0<t<1$ then follows easily from the $t=1$ case, the identity $D^{t}=D^{1} * I^{1-t}$, and Theorem $1 \cdot 3(a)$.

A theorem of Prawitz [10, theorem 3.16] says that $\mathscr{U} \subset H^{p}$ for all $p<\frac{1}{2}$, and hence $\mathscr{U} \subset A^{p}$ for all $p<1$ (the Koebe function shows that a univalent function need not be in $A^{1}$ ). Thus Proposition $5 \cdot 1$ implies that $D^{t} f \in A^{q}$ for all $q<2 /(2+t)$. Alternatively, this fact follows in the case $t=1$ from (the less subtle part of) a wellknown result of Brennan [7] which says that if $f \in S$ then $\left|f^{\prime}\right|^{q}$ is integrable on the unit disc for all $-1-\epsilon<q<\frac{2}{3}$ as long as $\epsilon>0$ is suitably small; the case $0<t<1$ then follows as before.

Proposition 5.2. If $f \in \mathscr{U}$ and $0<t \leqslant 1$ then $D^{t} f \in A^{q}$, for any $q<2 /(t+2)$. The statement is false for $q=2 /(t+2)$. 
Proof. The first statement has been proved in the above paragraph, so we need only show that the exponent $q=2 /(t+2)$ cannot be achieved. For $t=1$ (i.e. $\left.q=\frac{2}{3}\right)$, an explicit calculation shows that the Koebe function $k(z)=z /(1-z)^{2}$ is a counterexample. For $0<t<1$, we have $D^{t} k=D^{1+t}$, and so $D^{t} k \notin A^{2 /(t+2)}$ by Lemma $3 \cdot 8$.

\section{REFERENCES}

[1] P. Ahern and M. Jevtić. Duality and multipliers for mixed norm spaces. Michigan Math. J. 30 (1983), 53-64.

[2] J. M. Anderson. Coefficient multipliers and solid spaces. J. Analysis 1 (1993), 13-19.

[3] J. M. Anderson and D. Girela. Inequalities of Littlewood-Paley type, multipliers and radial growth of the derivative of analytic functions. J. reine angew. Math. 465 (1995), 11-40.

[4] J. M. Anderson and A. L. Shields. Coefficient multipliers of Bloch functions. Trans. Amer. Math. Soc. 224 (1976), 255-265.

[5] S. AxLER. Bergman spaces and their operators; in Survey of some recent results in operator theory, Vol. 1 (eds. J. B. Conway and B. B. Morrel), Pitman Research Notes in Mathematics, vol. 171 (Longman Scientific \& Technical, 1988), pp. 1-50.

[6] O. BLasco. Multipliers on spaces of analytic functions. Can. J. Math. 47 (1995), 44-64.

[7] J. E. Brennan. The integrability of the derivative in conformal mapping. J. London Math. Soc. 18 (1978), 261-272.

[8] S. Buckley, M. S. Ramanujan and D. Vukotić. Bounded and compact coefficient multipliers between Bergman and Hardy spaces; Integral Equations Operator Theory, to appear.

[9] D. Campbell and R. Leach. A survey of $H^{p}$ multipliers as related to classical function theory. Complex Variables 3 (1983), 85-111.

[10] P. L. Duren. Theory of $H^{p}$ spaces (Academic Press, 1970).

[11] P. L. Duren and A. L. Shieldos. Coefficient multipliers of $H^{p}$ and $B^{p}$ spaces. Pacific J. Math. $32(1970), 69-78$.

[12] P. L. Duren and G. D. Taylor. Mean growth and coefficients of $H^{p}$ functions. Illinois $J$. Math. 14 (1970), 419-423.

[13] T. Flett. Some theorems on fractional integrals. Math. Proc. Camb. Phil. Soc. 55 (1959), $31-50$.

[14] T. FletT. Mean values of power series. Pacific J. Math. 25 (1968), 463-494.

[15] T. FLETT. The dual of an inequality of Hardy and Littlewood and some related inequalities. J. Math. Anal. Appl. 38 (1972), 746-765.

[16] G. H. HaRdy and J. E. LitTtLwood. Some properties of fractional integrals, II. Math. Z. 34 (1932), 403-439.

[17] C. A. Horowitz. Zeros of functions in the Bergman spaces. Duke. J. Math. 41 (1974), 693-710.

[18] M. Jevtić and I. Jovanović. Coefficient multipliers of mixed norm spaces. Canad. Math. Bull. 36 (1993), 283-285.

[19] M. Jevtić and M. Pavlović. On multipliers from $H^{p}$ to $l^{q}, 0<q<p<1$. Arch. Math. 56 (1991), 174-180.

[20] P. Koskela. An inverse Sobolev lemma. Rev. Mat. Iberoamericana 10 (1994), 123-141.

[21] C. N. Kellogg. An extension of the Hausdorff-Young theorem. Michigan Math. J. 18 (1971), $121-127$.

[22] D. Luecking. Embedding theorems for spaces of analytic functions via Khinchine's inequality. Michigan Math. J. 40 (1993), 333-358.

[23] J. E. Littlewood and R. E. A. C. Paleey. Theorems on Fourier series and power series (II). J. London Math. Soc. 42 (1931), 52-89.

[24] J. Marsden. Basic complex analysis (W. H. Freeman, 1972).

[25] M. Mateluević and M. Pavlović. $L^{p}$ behaviour of the integral means of analytic functions. Studia Math. 77 (1984), 219-237.

[26] M. Mateljević and M. Pavlović. Multipliers of $H^{p}$ and BMOA. Pacific J. Math. 146 (1990), $71-84$.

[27] A. Nakamura, F. Ohya and H. Watanabe. On some properties of functions in weighted Bergman spaces. Proc. Fac. Sci. Tokai Univ. 15 (1979), 33-44.

[28] M. Pavlović. Mixed norm spaces of analytic and harmonic functions II. Publ. Inst. Math. Beograd 41 (55) (1987), 97-110.

[29] D. Vuкотіс́. On the coefficient multipliers of Bergman spaces. J. London Math. Soc. 50 (1994), $341-348$. 
Fractional integration, differentiation, and weighted Bergman spaces 385

[30] P. WoJTAszczyк. On multipliers into Bergman spaces and Nevanlinna class. Canadian Math. Bull. 33 (1990), 151-161.

[31] A. Zabulionis. Differential operator in spaces of analytic functions. Liet. Mat. Rinkinys 24 (1984), 53-59. (English translation in Lithuanian Math. J. 1984, 32-36).

[32] K. ZHU. Duality of Bloch spaces and norm convergence of Taylor series. Michigan Math. J. 38 (1991), 89-101. 